\title{
Stress físico do árbitro de futebol no jogo
}

\author{
A. Rebelo \\ S. Silva \\ N. Pereira \\ J. Soares
}

Faculdade de Ciências do Desporto e de Educação Física Universidade do Porto

\section{RESUMO}

Só conhecendo com rigor as exigências que se colocam ao árbitro em jogo poderemos programar correctamente o seu treino. No presente trabalho caracterizou-se a actividade física realizada por árbitros portugueses em jogos de futebol da Primeira Liga e avaliou-se o respectivo impacto fisiológico. Constituíram a amostra deste trabalho 8 árbitros de futebol ( $37 \pm 6,6$ anos) pertencentes à $1^{\text {a }}$ Categoria Nacional.

O árbitro de futebol gasta mais de $60 \%$ do tempo de jogo em actividades de baixa a média intensidade (marcha: $33.4 \%$; trote: 25,9\%; corrida média: $3,4 \%$ ) o que corresponde a cerca de uma hora. A frequência cardíaca média em jogo é de $150 \pm 21.9$ bat. $\min ^{-1}$, o que corresponderá a cerca de $82 \%$ da FC máx. O árbitro realiza num jogo 10 a 15 sprints de 4 segundos. As principais conclusões deste estudo com repercussões para o treino do árbitro de futebol são as seguintes: (i) os sprints devem ter uma duração inferior a 6 segundos ( \pm 30 metros) (ii) dado serem as capacidades velocidade e resistência duas das capacidades motoras mais solicitadas em jogo, na avaliação física do árbitros aconselha-se a utilização de um teste de velocidade (ex. velocidade aos 30 metros) e de um teste de resistência intermitente (ex. Yo-Yo endurance intermittent test).

Palavras-chave: futebol, caracterização do esforço, análise da frequência cardíaca, treino do árbitro

\section{ABSTRACT \\ Physical activity of soccer referees during the match}

To design a correct plan of physical training for soccer referees it is necessary to know the physical demands of the game. In this study, we characterise the physical activity performed by Portuguese referees during matches of the Portuguese National $1^{\text {st }}$ League, as well as the corresponding physiological impact, by means of continuous heart rate monitoring. This study included 8 referees $(37+6.6 \mathrm{yrs})$, of the $1^{\text {st }}$ Portuguese Category.

The referee spends more than $60 \%$ of the time in activities of low and medium intensity (walking: 33.4; jogging: 25.9\%; cruising: 4.4\%) whose duration is approximately $1 \mathrm{~h}$. The mean heart rate in the match was 15021.9 beats/min, corresponding to near $82 \%$ of the maximal heart rate. The referee performed 10 to 15 sprints of 4 seconds each. The main conclusions, as well as some indications for training planning, are: (i) sprints should have a duration less than 6 seconds $(+30 \mathrm{~m})$; knowing that speed and endurance are the main physical demands of the soccer referee during a match, in the tests battery for evaluation of the referees physical capacity, we suggest an inclusion of a speed test (e.g.: speed in $30 \mathrm{~m}$ ) as well as a endurance test (eg: Yo-Yo endurance intermittent test)

Keywords: soccer, physical profile, heart-rate analysis, referee conditioning 


\section{INTRODUÇÃO}

O árbitro de futebol é a entidade oficial que regula o comportamento desportivo dos intervenientes directos no jogo, fazendo cumprir as regras do jogo. Uma eficaz condução do jogo exige do árbitro uma boa percepção dos lances para uma correcta tomada de decisões (16). O árbitro deve agir com firmeza mantendo, tanto quanto possível, uma atitude discreta. No entanto, para que o árbitro tome as decisões mais ajustadas deve estar no local mais adequado para melhor apreciar as jogadas (6). O árbitro deve seguir as jogadas independentemente da intensidade dos movimentos dos jogadores e das alterações de ritmo do próprio jogo. Assim, exige-se que o árbitro esteja fisicamente bem preparado, que possua conhecimentos técnicos relativos às regras e que tenha os atributos psicológicos para enfrentar a imensa variedade de situações e experiências em que o jogo de futebol é fértil $(11,17)$.

É opinião relativamente consensual, tendo já sido alvo de numerosos estudos, que as exigências físicas que se colocam aos jogadores de futebol têm vindo a aumentar nas últimas décadas em resultado do aumento do ritmo do jogo (para refs ver 12, 13). A aptidão física que se exige ao árbitro de hoje está também condicionada por este aumento do ritmo do jogo. Ou seja, cremos que jogos com ritmo mais intenso exigirão do árbitro uma melhor aptidão física para estar no local certo no momento certo. Por outro lado, os árbitros de competições profissionais são normalmente cerca de 15 anos mais velhos do que os jogadores e têm uma actividade profissional para além da arbitragem (16), facto que nos faz admitir que a taxa de trabalho dos árbitros deve ser considerada como apreciável.

Apesar do importante papel ocupado pelo árbitro no jogo de futebol, a investigação na área da arbitragem é reduzida (9). Com efeito, apesar de ser frequente a investigação sobre a performance e o treino dos jogadores e equipas, a investigação sobre os factores da performance e do treino do árbitro não é muito abundante $(4,8,10,11)$. Situando a análise nas exigências físicas e fisiológicas que se colocam ao árbitro durante o jogo, a arbitragem em futebol é, em termos globais, uma actividade intermitente com uma intensidade apreciável. Catterall et al. (3) encontraram em árbitros de futebol valores médios da fre- quência cardíaca (FC) de 165 bat.min ${ }^{-1}$. Outros autores $(4,7,8)$, encontraram valores sobreponíveis para a FC durante o jogo. Estes valores corresponderão a cerca de $85-90 \%$ da FC máx. $(4,6,8)$. Para Harley et al. (6) e Johnston \& McNaughton (7) a fonte de energia mais solicitada pelo árbitro durante o jogo é o metabolismo aeróbio. No entanto, deve ser concedido ao metabolismo anaeróbio um papel central para a performance do árbitro, dada a relação entre a resistência em exercício intermitente de elevada intensidade e a diminuição da distância entre o árbitro e o local do terreno de jogo em que a infracção às leis do jogo ocorre (8).

A arbitragem em futebol parece ser, por isso, uma actividade que exige uma elevada taxa de trabalho físico, pelo que se justifica desenvolver metodologias de treino específicas para os árbitros, bem como recorrer a testes que permitam uma rigorosa avaliação da sua capacidade física. Ou seja, os árbitros devem seguir uma preparação física adequada à dinâmica que lhes é específica. O desenvolvimento da aptidão física do árbitro permitir-lhe-á estar suficientemente próximo das jogadas para um melhor controlo do jogo, seja qual for o ritmo imposto pelas equipas. Sabe-se que a fadiga física contribui para aumentar a probabilidade de erro provocando também a diminuição da capacidade de decisão (16). No presente trabalho pretendeu-se caracterizar a actividade física realizada por árbitros portugueses em jogos de futebol da Primeira Liga bem como, através da análise da FC, avaliar o respectivo impacto fisiológico. Trata-se, portanto, de um estudo baseado na avaliação física e fisiológica do árbitro em situação real de jogo.

\section{Material e métodos}

Constituíram a amostra deste trabalho 8 árbitros portugueses de futebol ( $37 \pm 6,6$ anos) pertencentes à $1^{\text {a }}$ Categoria Nacional. Quanto à experiência profissional, os árbitros estudados encontravam-se nesta categoria há 7,25 $\pm 3,9$ anos.

A recolha de dados realizou-se em 8 jogos da época 2000-01 (6 jogos da $1^{\text {a }}$ Liga de Futebol Portuguesa, um jogo da $2^{\text {a }}$ Liga de Futebol Portuguesa e um jogo dos quartos de final da Taça de Portugal). Foi, portanto, analisado um jogo de cada árbitro.

A FC foi monitorizada durante todo o jogo através 
de um aparelho de rádio-telemetria de baixo alcance (Polar Vantage NV, Kempele, Finland) com registo da FC de 5 em 5 segundos.

A actividade física realizada durante o jogo foi caracterizada através de um Estudo de Tempo e Movimento utilizando a metodologia sugerida por Reilly e Thomas (14) e constou dos seguintes passos:

1. Filmagem dos jogos - a câmara de filmar foi colocada num ponto médio em relação à dimensão longitudinal do campo e o mais alto possível. Cada árbitro foi filmado individualmente durante todo o jogo, incluindo as interrupções do mesmo;

2. Medição da duração dos deslocamentos - a partir da gravação em vídeo da movimentação do árbitro no jogo foi medido o tempo gasto em cada deslocamento com o auxílio do cronómetro integrado no vídeo;

3. Tipos de deslocamento - foram considerados sete tipos de deslocamento de acordo com a sua forma e intensidade: parado, marcha, trote, corrida média, sprint, deslocamentos de costas e deslocamentos laterais. A intensidade de deslocamento foi determinada subjectivamente com o auxílio de dois indicadores: a frequência gestual (i.e., número de movimentos/unidade de tempo) e a imagem de esforço apresentada pelo árbitro.

Foram utilizadas a medidas descritivas média, desvio-padrão e percentagens. Para comparação de resultados das duas parte do jogo foi utilizado teste Wilcoxon 2 related samples $(\mathrm{p}<0.05)$.

\section{Resultados e discussão}

No Quadro 1 e na Figura 1 são apresentados os valores da duração dos diversos tipos de deslocamento realizado pelo árbitro durante o jogo de futebol.
Quadro 1 - Média (X), desvio padrão (SD) e percentagens [\%] da duração (minutos) de cada tipo de deslocamento realizado pelo árbitro no jogo.

\begin{tabular}{|c|c|c|c|}
\hline & \multicolumn{3}{|c|}{ Duração } \\
\hline & $1^{\text {a }}$ Parte & $2^{\mathrm{a}}$ Parte & Jogo Completo \\
\hline Parado & $7.4 \pm 1.3$ & $9.1 \pm 1.7^{*}$ & $16.6 \pm 2.8(17.1 \%)$ \\
\hline Marcha & $15.3 \pm 2.1$ & $16.9 \pm 1.8$ & $32.3 \pm 2.7(33.4 \%)$ \\
\hline Trote & $13.3 \pm 1.0$ & $11.8 \pm 2.2$ & $25.1 \pm 2.3(25.9 \%)$ \\
\hline Corrida Média & $1.4 \pm 0.5$ & $1.8 \pm 0.5$ & $3.3 \pm 0.6(3.4 \%)$ \\
\hline Sprint & $0.3 \pm 0.2$ & $0.5 \pm 0.2$ & $0.8 \pm 0.2(0.8 \%)$ \\
\hline Desloc. Lado & $0.8 \pm 0.5$ & $0.9 \pm 0.6$ & $1.7 \pm 1.0(1.8 \%]$ \\
\hline $\begin{array}{l}\text { Desloc. Costas } \\
*_{-}^{*} p<0.05\end{array}$ & $8.5 \pm 1.4$ & $8.7 \pm 1.9$ & $17.1 \pm 2.6(17.7 \%)$ \\
\hline
\end{tabular}

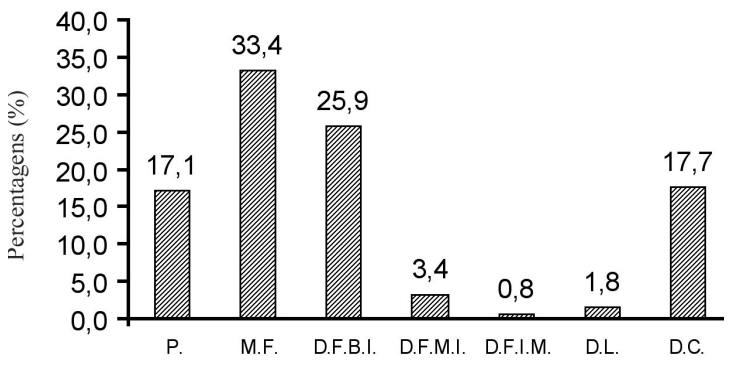

Figura 1 - Valores percentuais da duração tota de cada tipo de deslocamento realizado no jogo. P-parado; M.F. - marcha; DFBI - trote; DFMI - corrida média; $D F I M$ - sprint; $D L$ - deslocamentos laterais; $D C$-deslocamentos de costas.

Conforme pode ser constatado no Quadro 1, os árbitros ficam mais tempo parados na segunda parte do que na primeira parte dos jogos $(\mathrm{p}<0.05)$. A fadiga acumulada na segunda parte do jogo pode ser sugerida como uma das razões mais óbvias para este resultado. Nos outros tipos de deslocamento não foram encontradas diferenças com significado estatístico entre as duas partes do jogo.

Um dos dados de maior relevo a retirar do Quadro 1 e da Figura 1 refere-se aos deslocamentos de costas (DC). Comparando o tempo gasto neste tipo de deslocamento pelo árbitro e por jogadores de futebol (13) constatamos que o árbitro realiza uma superior percentagem de deslocamentos deste tipo (árbitro $17,1 \%$; jogador $7,7 \%$ ) . Num recente trabalho de Krustrup e Bangsbo (8) em que foram estudados 
árbitros dinamarqueses de elite verificou-se que o tempo que os árbitros gastavam em deslocamentos de costas ocupava apenas $5 \%$ do tempo total de jogo. De todo o modo, não devemos deixar de relevar o tempo gasto pelos árbitros portugueses em deslocamentos de costas. Ou seja, estes dados revelam a importância que o árbitro deve conceder a este tipo de deslocamentos no seu programa de treino. Os resultados deste estudo mostram que o sprint é o tipo de deslocamento em que os árbitros gastam menos tempo (0.8\%). Krustrup e Bangsbo (8) referem um valor inferior $(0.5 \%)$. Este dado não significa de modo algum que se trate de um deslocamento a negligenciar no treino do árbitro. Mais a diante retomaremos este ponto.

Podemos ainda observar na Figura 1 que o árbitro de futebol gasta mais de $60 \%$ do tempo de jogo em actividades de baixa e média intensidade (marcha: $33.4 \%$; trote: $25,9 \%$; corrida média: $3,4 \%$ ) o que corresponde a cerca de 60 minutos do tempo total de jogo. O árbitro pode percorrer no jogo cerca de 10 quilómetros: $9.5 \mathrm{~km}$ em árbitros da Premier Division English League (16); $11.2 \mathrm{~km}$ em árbitros da Liga de Futebol Profissional Japonesa (1); 10.1 km em árbitros da Primeira Liga Dinamarquesa (8). Ou seja, o árbitro pode percorrer cerca de $10 \mathrm{~km}$ no jogo realizando exercício de baixa a média intensidade em cerca de dois terços do tempo de jogo.

No Quadro 2 e Figura 2 são apresentados os valores da duração de cada deslocamento realizado pelos árbitros no jogo.

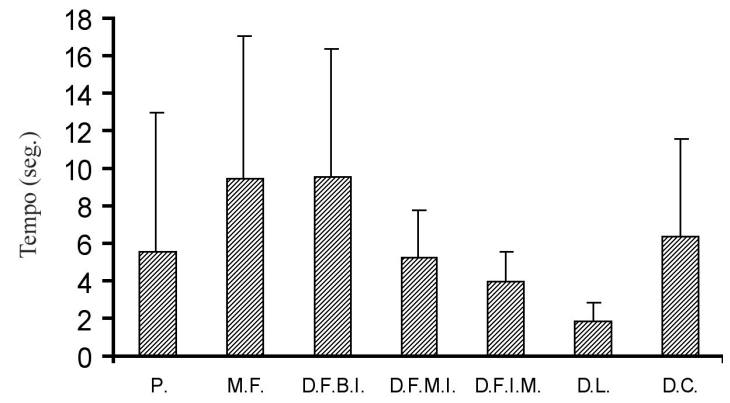

Figura 2 - Valores médios da duração (segundos) de cada deslocamento. $P$ - parado; M.F. - marcha; DFBI - trote; DFMI - corrida média; DFIM - sprint, $D L$ - deslocamentos laterais; $D C$ - deslocamentos de costas.

Um dos primeiros dados a retirar da análise da Figura 2 é o elevado desvio-padrão da duração de cada tipo de deslocamento, o que reflecte a variabilidade da duração das acções e fases do próprio jogo. Em segundo lugar, sobressai da análise deste gráfico a brevidade da generalidade dos deslocamentos, em particular dos sprints (DFIM). Com efeito, um dado importante a extrair destes resultados refere-se à duração dos sprints (cerca de 4 segundos), aliás dado idêntico ao encontrado em futebolistas portugueses no campeonato nacional da $1^{\text {a }}$ divisão (13). Na Figura 3 apresenta-se a distribuição da duração dos sprints realizados pelo árbitro no jogo por intervalos de frequência, para melhor conhecermos as características da duração destes deslocamentos.

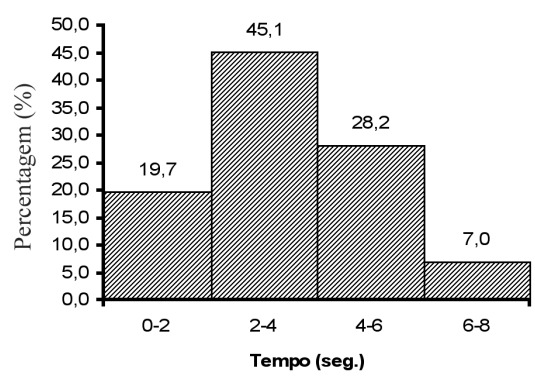

Figura 3 - Distribuição de frequências da duração dos sprints realizados pelos árbitros no jogo. 
Como podemos constatar na Figura 3, mais de 90\% dos sprints realizados pelo árbitro no jogo têm uma duração inferior a 6 segundos. Assim, no treino de velocidade do árbitro de futebol, aconselha-se usar estímulos de breve duração (2-6 segundos), o que corresponderá a distâncias inferiores a 30 metros. Um outro dado que deve levar-se em linha de conta na programação do treino do árbitro é a frequência dos deslocamentos. No Quadro 3 e na Figura 4 são mostradas as frequências dos diversos tipos de deslocamento realizados pelo árbitro no jogo.

Quadro 3 - Valores médios das frequências de cada deslocamento realizado pelo árbitro em no jogo, na $1^{a}$ e na $2^{a}$ parte.

\begin{tabular}{lccc} 
& \multicolumn{3}{c}{ Frequências } \\
& $1^{\text {a Parte }}$ & $2^{\text {a Parte }}$ & Amostra global \\
\hline Parado & $84.5 \pm 15.4$ & $91.8 \pm 10.4$ & $176.0 \pm 23.1$ \\
Marcha & $100.0 \pm 6.9$ & $103.3 \pm 10.5$ & $203.3 \pm 17,8$ \\
Corrida lenta & $78.0 \pm 8.8$ & $78.5 \pm 4.9$ & $155.0 \pm 14.2$ \\
Corrida média & $16.3 \pm 5.0$ & $20.8 \pm 5.3$ & $37.1 \pm 9.9$ \\
Sprint & $4.3 \pm 2.3$ & $7.5 \pm 2.6^{*}$ & $11.8 \pm 4.4$ \\
Deslocam. lado & $26.7 \pm 18.2$ & $16.5 \pm 7.0$ & $54.8 \pm 33.8$ \\
Deslocam. costas & $78.2 \pm 13.9$ & $82.3 \pm 13.4^{*}$ & $143.8 \pm 66.6$ \\
$*_{-}^{*}$ p<0.05 & & & \\
& & &
\end{tabular}

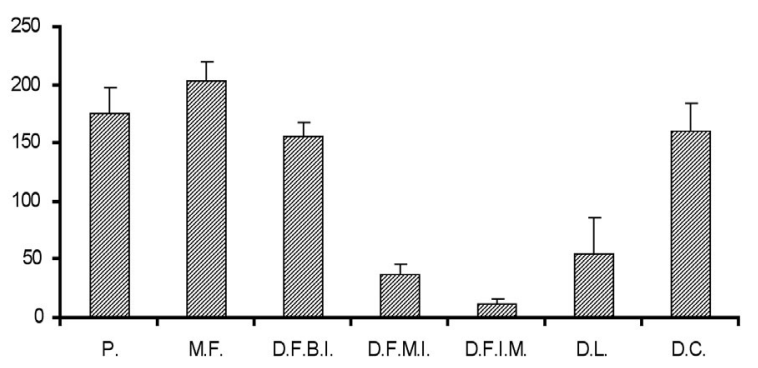

Figura 4 - Frequência de cada tipo de deslocamento realizado pelo árbitro no jogo.

P - parado; M.F. - marcha; DFBI - trote; DFMI - corrida média; DFIM - sprint. $D L$ - deslocamentos laterais; $D C$-deslocamentos de costas.

Em primeiro lugar registe-se o número de sprints realizados pelo árbitro no jogo (10-15). Não sendo um número muito elevado (os futebolistas realizam cerca de 80-90 sprints por jogo, 13), não devemos atribuir-lhes um papel secundário, dada a importância que este tipo de deslocamentos têm na performance do árbitro. Com efeito, trata-se de deslocamentos muitas vezes associados a acções de muita importância no desenrolar do jogo, como por exemplo as acções de contra-ataque; acções que no futebol estão fortemente ligadas à possibilidade de ocorrência de jogadas com grande probabilidade de golo.

$\mathrm{Na}$ segunda parte do jogo os árbitros realizaram mais sprints e mais deslocamentos de costas $(\mathrm{p}<0.05)$. Estes resultados contrariam a ideia de que a fadiga acumulada possa induzir uma diminuição da quantidade de exercício de alta intensidade realizado na segunda parte do jogo.

Analisemos agora o comportamento da FC dos árbitros estudados durante o jogo (Quadro 4).

Quadro 4 - Valores médios da FC dos árbitros durante o jogo.

\begin{tabular}{lccc} 
Árbitro & FC $1^{\text {a }}$ parte & FC $2^{\text {a }}$ parte & FC jogo completo \\
\hline 1 & $161 \pm 12,1$ & $153 \pm 11,9$ & $157 \pm 12,6$ \\
2 & $136 \pm 12,5$ & $134 \pm 13,4$ & $135 \pm 13,0$ \\
3 & $134 \pm 13,1$ & $127 \pm 13,1$ & $130 \pm 13,5$ \\
4 & $140 \pm 13,9$ & $139 \pm 14,2$ & $139 \pm 14,1$ \\
5 & $184 \pm 8,6$ & $188 \pm 7,9$ & $186 \pm 8,5$ \\
6 & $145 \pm 13,4$ & $148 \pm 10,7$ & $146 \pm 12,3$ \\
7 & $181 \pm 9,0$ & $173 \pm 8,8$ & $177 \pm 9,7$ \\
8 & $129 \pm 12,2$ & $126 \pm 14,1$ & $127 \pm 13,3$ \\
Amostra global & $151 \pm 21,5$ & $148 \pm 22,2$ & $150 \pm 21,9$
\end{tabular}

$\mathrm{O}$ valor médio da FC média que encontramos no nosso estudo $\left(150 \pm 21.9\right.$ bat. $\left.\mathrm{min}^{-1}\right)$ é ligeiramente mais baixo do que os encontrados na literatura (162 bat.min ${ }^{-1}, 7 ; 165$ bat.min $\left.{ }^{-1}, 3\right)$. É provável que esta diferença de resultados se deva aos diferentes ritmos de jogo característicos dos campeonatos de cada país. Tanto o campeonato dinamarquês como o campeonato inglês são campeonatos em que o ritmo de jogo é muito intenso e superior ao ritmo de jogo identificado no campeonato português. Esta ideia é ilustrada pelo superior tempo gasto em corrida (trote, corrida média e sprint) pelos jogadores do campeonato dinamarquês quando comparados com os do campeonato português (35 minutos, 2 vs 32 
minutos, 13 , respectivamente). Um quadro semelhante é encontrado na distância percorrida em corrida nos campeonatos inglês e português (7264 metros, 14 vs 6285 metros, 13).

No Quadro 5 são apresentados os valores médios da FC máx. atingida pelos árbitros durante o jogo.

Quadro 5 - Valores médios da FC máx. dos árbitros durante o jogo.

\begin{tabular}{llllllllll} 
Árbitro & 1 & 2 & 3 & 4 & 5 & 6 & 7 & 8 & Amostra global \\
\hline
\end{tabular}
FC máx. $181165160177202174197156 \quad 176 \pm 17$

Segundo Krustrup e Bangsbo (8) o valor mais alto da FC que um árbitro atinge durante o jogo corresponde a cerca de $97 \%$ da sua FC máxima. O valor médio da FC máx. atingida em jogo no nosso estudo foi de $176 \pm 17$ bat.min ${ }^{-1}$, o que corresponderá, de acordo com o estudo citado, a uma FC máxima estimada de $181 \pm 17$ bat. $\mathrm{min}^{-1}$. Se usarmos este valor estimado para a FC máx. e o relacionarmos com a FC média encontrada no nosso estudo $(150 \pm 21.9$ bat. $\left.\mathrm{min}^{-1}\right)$, podemos dizer que os árbitros que estudamos realizaram os jogos a uma intensidade média de exercício de cerca de $82 \%$ da FC máx. No estudo atrás citado, a FC média dos árbitros em jogo foi de $85 \%$ da FC máx. o que nos sugere, mais uma vez, a inferior intensidade do exercício encontrada nos indivíduos da nossa amostra.

Um outro dado relevante dos valores médios da FC durante o jogo é a amplitude de valores em função dos árbitros avaliados (min: 127 bat.min ${ }^{-1}$; máx:186 bat. $\mathrm{min}^{-1}$ ). Na origem destas diferenças inter-individuais de valores da FC em jogo podem estar factores como a diferença etária (amplitude: 26-44 anos), os diferentes estilos de arbitragem e níveis diferentes de aptidão física. As diferenças de ritmo de jogo entre níveis de competição poderão também explicar estes resultados. Ekblom (5) num estudo do tipo Tempo e Movimento verificou que a intensidade do exercício era maior em jogos da primeira divisão do que em jogos da $2^{\text {a }}$ divisão. Partindo destes resultados e de outros dados fisiológicos observados em jogo o autor considerou mesmo que, sob o ponto de vista físico, aquilo que melhor distingue jogadores de diferente nível competitivo é a quantidade de exercício de elevada intensidade realizada em jogo; quanto maior o nível competitivo mais elevada a quantidade de exercício de elevada intensidade realizado no jogo.

Idealmente, é desejável que o árbitro realize o seu trabalho de forma económica, isto é, com o menor esforço possível, já que como foi atrás referido a fadiga pode interferir na qualidade da arbitragem. Com efeito, a realização de exercício a uma intensidade superior a $50 \% \mathrm{VO}_{2}$ máx. pode induzir a diminuição das funções cognitiva e psicomotora (15). Uma das estratégias mais eficazes para melhorar a aptidão física é, obviamente, desenhar programas de treino que elevem o grau de preparação física especifica do árbitro.

\section{Em síntese}

1. exige-se ao árbitro de futebol aptidão para realizar exercício intermitente de média intensidade e prolongado; uma boa resistência em exercício intermitente deve fazer parte dos principais objectivos de treino físico do árbitro;

2. o treino de velocidade deve também ser incluído nos conteúdos de treino físico do árbitro.

Apresentamos, por último, no Quadro 6 uma sugestão de princípios metodológicos para o treino físico do árbitro, em particular para o treino da velocidade e para o treino da resistência para o exercício intermitente de alta intensidade.

Quadro 6 - Princípios metodológicos para o treino da velocidade e da resistência para o exercício intermitente de alta intensidade de árbitros de futebol.

\begin{tabular}{|c|c|c|c|}
\hline \multirow[b]{2}{*}{ Intensidade } & \multicolumn{3}{|c|}{ Velocidade } \\
\hline & $\begin{array}{l}\text { Duração } \\
\text { exercício }\end{array}$ & $\begin{array}{l}\text { Duração } \\
\text { repouso }\end{array}$ & $\begin{array}{c}\text { Número } \\
\text { repetições }\end{array}$ \\
\hline Máxima & 2-6 seg. & $20-60$ seg. & $4-8$ \\
\hline
\end{tabular}

\begin{tabular}{lccc}
\multicolumn{2}{c}{ Resistência para o exercício intermitente de alta intensidade } \\
\begin{tabular}{lccc} 
Intensidade & Duração & Duração & Número \\
& exercício & repouso & repetições \\
\hline Média/Alta & $10-20$ seg. & $60-90$ seg. & $6-10$
\end{tabular}
\end{tabular}




\section{CORRESPONDÊNCIA}

\section{António Natal Campos Rebelo}

Faculdade de Ciências do Desporto

e de Educação Física

Universidade do Porto

Rua Dr. Plácido Costa, 91

4200.450 Porto

Portugal

anatal@fcdef.up.pt

\section{REFERÊNCIAS}

1. Asami T, Togari H, OHASHI J (1987). Analysis of movement patterns of referees during soccer matches. In: Reilly T, Lees A, Davies K, Murphy J (Eds.). First World Congress on Science and Football. New York: E. \& F.N. Spon, 341-346.

2. Bangsbo J, Norregard L, Thorso F (1991). Activity profile of competition soccer. Can J Sport Sci 16 (2): 110-116.

3. Catterall C, Reilly T, Atkinson G, Coldwells A (1993). Analysis of the work rates of association football referees. Br J Sports Med 27: 193-196.

4. D’Ottavio S, Castagna C (2001). Physiological load imposed on elite soccer referees during actual match play. $J$ Sports Med Phys Fitness 41: 27-32.

5. Ekblom B (1986). Applied physiology of soccer. Sports Med 3: 50-60.

6. Harley R, Tozer K, Doust J (1999). An analysis of movement patterns and physiological strain in relation to optimal positioning of Association Football Referees. J Sports Sci 17: 10 .

7. Johnston L, McNaughton L (1994). The physiological requirements of Soccer refereeing. Aust J Sci Med Sport 26: 67-72.

8. Krustrup P, Bangsbo J (2001). Physiological demands of top class soccer refereeing in relation to physical capacity: effect of intense intermittent exercise training. J Sports Sci 19: 881-891.

9. Manson C, Lovell G (2001). An examination of the perceived demands, attitudes and self-reported preparation of referees in the English Football Association Premier League. Communications of the annual conference of the British Association of Sport and Exercise Sciences. J Sport Sci 19 (1): 24.

10. Martin J, Tolfrey K, Smith N, Jones, A (2001). Match analysis of Premiership rugby union football refereeing. Communications of the annual conference of the British Association of Sport and Exercise Sciences. J Sport Sci 19: 23-24.

11. Neto J (1999). A Preparação Física e Psicológica do Árbitro de Futebol. Porto: Edições ASA.

12. Rebelo A, Soares JMC (1992). A comparative study of time-motion analysis during the two halves of a soccer game. Proceedings of the First World Congress of Notational Analysis of Sport. Liverpool

13. Rebelo A (1993). Caracterização da actividade física de futebolistas em competição. Provas de Aptidão pedagógica e de Capacidade Científica. Porto: ISEF - Universidade do Porto.

14. Reilly T, Thomas V (1976). A motion analysis of work-rate in different positional roles in professional football matchplay. J Human Mov Studies 2: 87-97.

15. Reilly T, Smith D (1986). Effect of work intensity on performance in a psychomotor task during exercise. Ergonomics 29: 601-606.

16. Reilly T (1996). Special populations. In: Reilly, T. (Ed.). Science and Soccer. London: E \& F.N. Spon.

17. Taylor A, Daniel J (1988). Sources of Stress in Soccer Officiating: an empirical study. In: Reilly T, Lees A, Davids K, Murphy W (Eds.). Science and Football. London: E. \& F.N. Spon. 